\title{
Active vibration suppression of a beam using piezoeffect
}

\author{
Nelly Rogacheva ${ }^{*}$ \\ Moscow State University of Civil Engineering, Yaroslavskoe shosse, 26, Moscow, 129337, Russia
}

\begin{abstract}
Operation of structures and equipment in dynamic conditions led to the problems of vibration isolation and vibration suppression. For vibration isolation and vibration suppression passive, active systems and their combinations are used. Passive vibration isolation usually consists in the fact that the protected object relies on extremely dimensional springs and vibration isolators. Vibration isolation systems containing only passive elastic and damping elements are called passive. Active vibration isolation and vibration damping systems use external energy sources. These are pneumatic, hydropneumatic and hydromechanical devices. Recently, electro-elastic and magneto-elastic systems [1], [2] began to be used for vibration isolation and active vibration suppression. As a rule, the analysis of the work of such systems consists in the development of an experimental layout and a schematic diagram. In this paper, a mathematically based model is used to solve the problem in question. The calculations are performed and the results are presented in the form of graphs.
\end{abstract}

\section{Introduction}

A three-layer beam with one elastic layer and two piezoelectric layers located symmetrically with respect to the elastic layer is considered. The middle layer is elastic; the outer layers are made of a piezoelectric material. The beam is related to Cartesian coordinates. The axis $x_{1}$ is directed along the length of the beam, the axis $x_{2}$ is directed along the width of the beam, the axis $x_{3}$ is orthogonal to them.

The axis $x_{1}$ is directed along the length of the beam, the axis $x_{2}$ is directed along the width of the beam.

It is assumed that the piezoelectric layers are pre-polarized in the direction $x_{3}$ [3] - [5].

Longitudinal section of the beam in Cartesian coordinates and the electrical load are schematically shown in Fig. 1.

\footnotetext{
* Corresponding author: nrogache@yandex.ru
} 


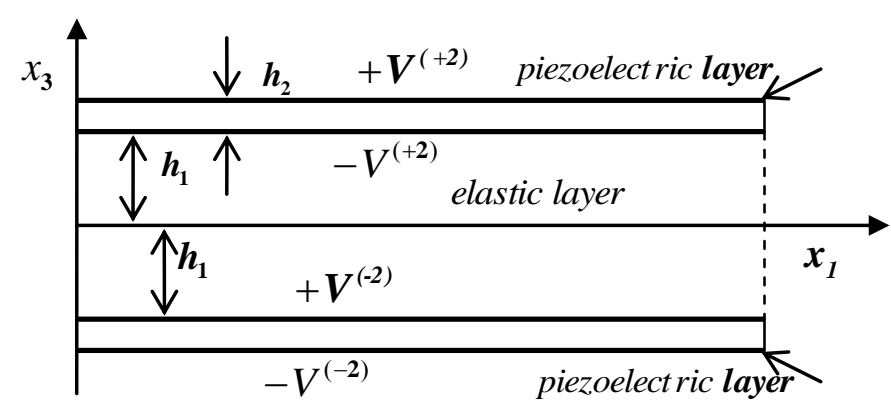

Fig. 1. Schematic representation of the structure of the layered beam.

For our purposes, we will consider piezoelectric layers, in which the faces $\mathrm{x}_{3}=$ const are completely covered with electrodes. If an electric potential $\varphi$ is specified on the electrodes, then the boundary conditions are

$$
\left.\varphi^{( \pm 2)}\right|_{x_{3}= \pm \boldsymbol{h}}= \pm V^{( \pm 2)} ;\left.\quad \varphi^{( \pm 2)}\right|_{x_{3}= \pm h_{1}}=\mp V^{( \pm 2)}
$$

On short-circuited electrodes, the electric potential is zero

$$
\left.\varphi^{( \pm 2)}\right|_{x_{3}= \pm \boldsymbol{h}}=\left.\varphi^{( \pm 2)}\right|_{x_{3}= \pm h_{1}}=\mathbf{0}
$$

The superscript in parentheses indicates the layer number. Hereinafter, each formula with double signs \pm , $\mp$ contains two formulas. To get one formula, you should take only the upper signs, to get the second formula you need to leave only the lower signs.

In [6], we obtain the elastic relations for a multilayer electroelastic beam. Here we briefly present these results for a particular case - a three-layer beam.

In the case of thin-walled beams in the equations of state, the stresses $\sigma_{22}$ and $\sigma_{33}$ can be neglected compared to the stresses $\sigma_{11}$. In addition, it is assumed that the electroelastic state does not depend on the coordinate $x_{2}$.

Taking into account the assumptions made, the equations for the elastic and electroelastic layers will be written as

The system of equilibrium equations takes the form

$$
\frac{\partial \sigma_{i i}^{(k)}}{\partial x_{i}}+\frac{\partial \sigma_{i j}^{(k)}}{\partial x_{j}}=\rho \frac{\partial^{2} u_{i}^{(k)}}{\partial t^{2}}, i \neq j=\mathbf{1 , 3}, k=-\mathbf{2 , 1 , 2}
$$

Strain - displacement formulas

$$
e_{\mathbf{1}}^{(k)}=\frac{\partial u_{\mathbf{1}}^{(k)}}{\partial x_{1}}, \boldsymbol{k}=-\mathbf{2 , 1 , 2}
$$

Equation of state (Hooke's law) for the elastic layer

$$
\sigma_{11}^{(1)}=E_{1} e_{1}^{(1)}
$$


Equations of state for piezoelectric layers

$$
\begin{aligned}
& \sigma_{11}^{( \pm 2)}=\frac{1}{s_{11}^{E}} e_{1}^{( \pm 2)}-\frac{d_{31}}{s_{11}^{E}} E_{3}^{( \pm 2)} \\
& D_{3}^{( \pm 2)}=\varepsilon_{33}^{T} E_{3}^{( \pm 2)}+d_{31} \sigma_{11}^{( \pm 2)}
\end{aligned}
$$

where

$$
E_{\mathbf{3}}^{( \pm \mathbf{2})}=-\frac{\partial \varphi^{( \pm \mathbf{2})}}{\partial x_{\mathbf{3}}}
$$

In formulas (3) - (8) $u_{1}$ and $e_{1}$ are the displacement and deformation in the direction $x_{1}$, respectively, $E_{3}$ and $D_{3}$ are the components of the electric field vector and electric induction vector in the direction $x_{3}, s_{11}^{E}$ is the elastic compliance at zero electric field, $d_{31}$ is the piezoelectric constant, $\varepsilon_{33}^{T}$ is the dielectric constant at zero voltages. The notation used is the same as that used in [5]

On the surfaces of the beam, the mechanical surface load is usually specified as

$$
\left.\sigma_{13}^{( \pm 2)}\right|_{x_{3}= \pm h}= \pm q_{1}^{ \pm},\left.\quad \sigma_{33}^{( \pm 2)}\right|_{x_{3}= \pm h}= \pm q_{3}^{ \pm}
$$

\section{Derivation of equations of electroelastic beam}

In order to construct a theory of electroelastic beams, one should accept some assumptions regarding electrical quantities. As in the construction of the theory of piezoelectric plates and shells [5], the content of accepted hypotheses depends on the electrical conditions on the surfaces of the piezoelectric layers. For piezoelectric layers, we accept assumptions that were substantiated by the asymptotic method for single-layer electroelastic plates and shells. For a piezoelectric layer with electrodated faces, the component of the electric induction vector $D_{3}$ normal to the faces does not depend on the thickness coordinate $x_{3}$

$$
D_{\mathbf{3}}^{( \pm 2)}=D_{\mathbf{3 , 0}}^{( \pm 2)}\left(x_{1}\right)
$$

The electric potential is a quadratic function of the thickness coordinate $x_{\mathbf{3}}$

$$
\varphi^{( \pm 2)}=\varphi_{, 0}^{( \pm 2)}+x_{3} \varphi_{, 1}^{( \pm 2)}+x_{3}^{2} \varphi_{, 2}^{( \pm 2)}
$$

If the electric potential is set on the electrodes (1), then formula (11) can be converted to

$$
\varphi^{( \pm 2)}=\mp V^{( \pm 2)}+\left(x_{3} \mp \boldsymbol{h}_{1}\right)\left(\frac{2 V^{( \pm 2)}}{h_{k}} \mp h_{2} \varphi_{, 2}^{( \pm)}\right)+\left(x_{3} \mp h z_{1}\right)^{2} \varphi_{, 2}^{( \pm 2)}
$$

Taking into account formulas (12) and (8), we get

$$
E_{3,0}^{( \pm 2)}=-\frac{2 V^{( \pm 2)}}{h_{2}} \pm\left(\boldsymbol{h}+h_{1}\right) \varphi_{, 2}^{( \pm 2)}, \quad E_{3,1}^{( \pm 2)}=-2 \varphi_{, 2}^{( \pm 2)}, \quad \varphi_{, 2}^{( \pm)}=\frac{d_{31}}{2 \varepsilon_{33}^{T}} \sigma_{11,1}^{( \pm 2)}
$$


where

$$
E_{3}^{( \pm 2)}=E_{3,0}^{( \pm 2)}+x_{3} E_{3,1}^{( \pm 2)}
$$

The mechanical quantities of any layer for which the Kirchhoff hypotheses are valid can be written as the following linear functions of the coordinate $x_{3}$

$$
u_{1}=u_{1,0}+x_{3} u_{1,1}, \quad e_{1}=\varepsilon+x_{3} \kappa, \quad \sigma_{11}^{( \pm 2)}=\sigma_{11,0}^{( \pm 2)}+x_{3} \sigma_{11,1}^{( \pm 2)}
$$

Here $\varepsilon$ and $\kappa$ are the components of the tangential and bending deformation of the midline of the beam, respectively

$$
\varepsilon=\frac{\partial u}{\partial x_{1}}, \kappa=\frac{\partial^{2} w}{\partial x_{1}^{2}}, u=\left.u_{1}\right|_{x_{3}=\mathbf{0}}, w=-\left.u_{3}\right|_{x_{3}=\mathbf{0}}
$$

Taking into account formulas (6) - (16), the equations of state for the piezoelectric layers can be rewritten as

$$
\sigma_{11,0}^{( \pm 2)}=\frac{1}{s_{11}^{E}} \varepsilon+\frac{2 d_{31}}{h_{2} s_{11}^{E}} V^{( \pm 2)} \mp \frac{\left(h+h_{1}\right) k_{31}{ }^{2}}{2 s_{11}^{E}\left(1-k_{31}^{2}\right)} \kappa, \sigma_{11,1}^{( \pm 2)}=\frac{1}{s_{11}^{E}\left(1-k_{31}{ }^{2}\right)} \kappa
$$

Let us analyze the hypotheses that we used to reduce the three-dimensional problem to the one-dimensional theory of beams. It should be emphasized that for the piezoelectric layers with and without electrodes, different hypotheses are used, with the result that different coefficients are obtained in the one-dimensional electroelasticity relations (17). In addition, for the electric potential, many authors mistakenly accept the linear law of variation in the thickness of the beam. Under the linear law for the electric potential, the quantity $E_{3}$ is constant and in our notation is equal to

$$
E_{3}^{( \pm 2)}=E_{3,0}^{( \pm 2)}=-\frac{2 V^{( \pm 2)}}{h_{2}}
$$

If an incorrect linear law for electric potential is adopted, then the formula for the stresses of the piezoelectric layer takes the form

$$
\sigma_{11,0}^{( \pm 2)}=\frac{1}{s_{11}^{E}} \varepsilon+\frac{2 V^{( \pm)}}{h_{2}} \frac{d_{31}}{s_{11}^{E}}, \sigma_{11,1}^{( \pm 2)}=\frac{1}{s_{11}^{E}\left(1-k_{31}^{2}\right)} \kappa
$$

Substituting formulas (18) and (19) into (7), taking into account that $D_{\mathbf{3}}^{( \pm 2)}=D_{\mathbf{3 , 0}}^{( \pm 2)}\left(x_{\mathbf{1}}\right)$ and equating the coefficients with the same degrees of the coordinate $x_{3}$, we obtain

$$
D_{3,0}^{( \pm 2)}=\varepsilon_{33}^{T} E_{3,0}^{( \pm 2)}+d_{31} \sigma_{11,0}^{( \pm 2)}, \sigma_{11,1}^{( \pm 2)}=\mathbf{0}
$$

As a result of using the linear law for the electric potential, a contradiction was obtained the second formula (20) in the bending problem does not make sense. This means that the linear law hypothesis for electric potential is, generally speaking, erroneous.

Integrating the stresses in thickness of the beam, we find the resulting tangential force $T$ and bending moment $G$ 


$$
T=\int_{-h}^{+h} \sigma_{11} d x_{3}, \quad G=-\int_{-h}^{+h} \sigma_{11} x_{3} d x_{3}
$$

Having integrated the equations of motion and the equations of state for each layer, we obtain one-dimensional equations for a three-layer electroelastic beam. The equations of the theory of layered electroelastic beams of a symmetric structure have exactly the same form as in the case of thermoelastic beams. The problem in question, as in the theory of elasticity, is divided into two problems - a plane problem and a bending problem.

Plane problem

$$
\begin{gathered}
\frac{\partial T}{\partial x_{1}}+X=\mathbf{2} h \rho \frac{\partial^{2} u}{\partial t^{2}}, \quad T=A \varepsilon+P, \quad \varepsilon=\frac{\partial u}{\partial x_{1}} \\
X=q_{1}^{+}+q_{1}^{-}, \quad A=\mathbf{2} h_{1} E+\frac{\mathbf{2} h_{2}}{s_{11}^{E}}, \quad P=\mathbf{2} \frac{d_{31}}{s_{11}^{E}}\left(V^{(2)}+V^{(-2)}\right), \quad \rho=\frac{1}{h}\left(\rho_{1} h_{1}+\rho_{2} h_{2}\right) .
\end{gathered}
$$

Bending problem

$$
\begin{gathered}
\frac{\partial N}{\partial x_{1}}+Z=2 h \rho \frac{\partial^{2} w}{\partial t^{2}}, N=\frac{\partial G}{\partial x_{1}}, G=M \kappa+Q, \kappa=\frac{\partial^{2} w}{\partial x_{1}^{2}} \\
Z=-\left(q_{3}^{+}+q_{3}^{-}\right), M=-\frac{\mathbf{2} h_{1}{ }^{3} E}{3}-\frac{\mathbf{2}\left(h^{3}-h_{1}{ }^{3}\right) \kappa}{\mathbf{3} s_{11}^{E}\left(1-k_{31}^{2}\right)}\left(1-\frac{\mathbf{3} h_{2}\left(h^{2}-h_{1}^{2}\right) k_{31}^{2}}{\mathbf{4}\left(h^{3}-h_{1}^{3}\right)}\right) \\
Q=-\frac{d_{31}}{s_{11}^{E}} \frac{h^{2}-h_{1}^{2}}{h_{2}}\left(V^{(2)}-V^{(-2)}\right)
\end{gathered}
$$

Here $N$ is the shear force

$$
N=-\int_{-h}^{+h} \sigma_{13} d x_{3}
$$

Both problems, up to constant coefficients, coincide with the corresponding problems of the theory of thermoelastic beams. To solve these problems we will use well-developed methods of the theory of elastic beam.

\section{Longitudinal vibrations of a three -layer beam}

As an example, we consider the damping of harmonic vibrations of a three-layer rod (all values vary according to the variable $t$ according to the law $\boldsymbol{e}^{-i \omega t}$ where the variable $t$ is the time), therefore we will write down all the equations and boundary conditions with respect to the amplitude values of the unknown quantities.

The upper and lower piezoceramic layers are located symmetrically with respect to the middle elastic layer. The thickness of the elastic layer is equal $2 h_{1}$, the thickness of each piezoelectric layer is equal to $h_{2}$, the length of the rod is $l$, the width of the beam is $g$ (Fig. 1). One edge of the beam is rigidly fixed, the other is free from fasteners.

The following electrical load excites longitudinal vibrations of the beam: 


$$
V^{(2)}=V^{(-2)}=V_{p}
$$

Let us turn to the dimensionless coordinates and the dimensionless sought quantities equations

$$
\begin{gathered}
\xi=\frac{\boldsymbol{x}}{\boldsymbol{l}}, \boldsymbol{u}_{*}=\frac{\boldsymbol{u}}{\boldsymbol{l}}, T_{*}=\frac{\boldsymbol{T}}{\boldsymbol{A}}, T_{*}^{(e)}=\frac{\boldsymbol{T}^{(e)}}{\boldsymbol{A}}, P_{*}=\frac{\boldsymbol{P}}{\boldsymbol{A}}, \boldsymbol{X}_{*}=\frac{\boldsymbol{X} \boldsymbol{l}}{\boldsymbol{A}} \\
\frac{d T_{*}}{d \xi}+X_{*}+\lambda_{1}^{2} u_{*}=\mathbf{0}, T_{*}=\varepsilon_{*}+P_{*}, \varepsilon_{*}=\frac{d u_{*}}{d \xi}, \lambda_{1}^{2}=\frac{2 h \rho \omega^{2} \boldsymbol{l}^{2}}{\boldsymbol{A}}
\end{gathered}
$$

where $\lambda_{1}^{2}$ is the dimensionless frequency parameter

The resolving equation is

$$
\frac{d^{2} u_{*}}{d \xi^{2}}+\lambda_{1}^{2} u_{*}+\boldsymbol{X}_{*}=\mathbf{0}
$$

Its solution is

$$
\begin{gathered}
u_{*}=c_{1} \sin \lambda_{1} \xi+c_{2} \cos \lambda_{1} \xi-\frac{1}{\lambda_{1}^{2}} \boldsymbol{X}_{*} \\
T_{*}=\lambda_{1}\left(c_{1} \cos \lambda_{1} \xi-c_{2} \sin \lambda_{1} \xi\right)+P_{*}
\end{gathered}
$$

Arbitrary integration constants $c_{1}$ and $c_{2}$ are determined from the conditions at the ends of the beam

$$
\left.u_{*}\right|_{\xi=\mathbf{0}}=\mathbf{0},\left.T_{*}\right|_{\xi=\mathbf{1}}=T_{*}^{(e)}
$$

Satisfying conditions (26), we get

$$
c_{1}=\frac{\sin \lambda_{1}}{\lambda_{1}{ }^{2} \cos \lambda_{1}} X_{*}-\frac{1}{\lambda_{1} \cos \lambda_{1}} P_{*}+\frac{1}{\lambda_{1} \cos \lambda_{1}} T_{*}^{(e)}, \quad c_{2}=\frac{1}{\lambda_{1}^{2}} X_{*}
$$

The natural frequencies are determined from the equation $\cos \lambda_{1}=\mathbf{0}$ and they are equal to $n \pi+\pi \mathbf{2}, n=\mathbf{0 , 1}, \mathbf{2}, \mathbf{3}, \ldots$

Consider different cases.

We consider vibrations under the action of the edge tangential force $T_{*}^{(e)}\left(\boldsymbol{X}_{*}=\mathbf{0}\right)$

$$
\left.u_{*}\right|_{\xi=\mathbf{0}}=\mathbf{0},\left.\quad T_{*}\right|_{\xi=\mathbf{1}}=T_{*}^{(e)}
$$

In this case from formula (27) we have

$$
c_{1}=-\frac{1}{\lambda_{1} \cos \lambda_{1}} P_{*}+\frac{1}{\lambda_{1} \cos \lambda_{1}} T_{*}^{(e)}, c_{2}=\mathbf{0}
$$


We choose the potential difference so that $\boldsymbol{P}_{*}=T_{*}^{(e)}$. Then from the formulas (28), (25) it follows that $u_{*} \equiv \mathbf{0}$ at all points of the beam. It is valid for any frequency of forced vibrations. The force $T_{*}$ remains no wound to zero. It is constant along the length of the beam $T_{*}=T_{*}^{(e)}$.

Then we consider vibrations under action the distributed tangential load.

Let the distributed tangential load $\boldsymbol{X}_{*}$ be non-zero and $T^{(e)}$ equals zero. In this case, the vibrations cannot be fully canceled, but the amplitude of vibrations can be significantly reduced by proper selection of the electrical load.

For dynamics with a small variability of the stress-strain state along the longitudinal coordinate $\xi$, it is possible to pick up the electrical load $P_{*}$ so that the greatest value of the displacement $u_{*}$ becomes zero. To determine the three unknown constants, we obtain the following system of three equations:

$$
\left.u_{*}\right|_{\xi=\mathbf{0}}=\mathbf{0},\left.\quad T_{*}\right|_{\xi=\mathbf{1}}=\mathbf{0},\left.\quad u_{*}\right|_{\xi=\mathbf{1}}=\mathbf{0}
$$

from which we will find:

$$
P_{*}=\frac{\boldsymbol{X}_{*}}{\lambda_{1} \sin \lambda_{1}}\left(1-\cos \lambda_{1}\right), \quad c_{1}=\frac{\boldsymbol{X}_{*}}{\lambda_{1}{ }^{2}} \frac{\mathbf{1}-\cos \lambda_{1}}{\sin \lambda_{1}}, \quad c_{2}=\frac{\boldsymbol{X}_{*}}{\lambda_{1}{ }^{2}}
$$

The computational results are plotted in Fig. $2\left(\lambda_{1}=\mathbf{1}\right)$.Hereinafter, the black and red lines depict the values of the stress-strain state without suppression vibration suppression and with suppression, respectively.

Black lines correspond to the displacements and the forces of the beam with short-circuited electrodes under the action of a mechanical load. Red line corresponds to these quantities under the action of a mechanical load and a specially selected electrical load reducing displacements.

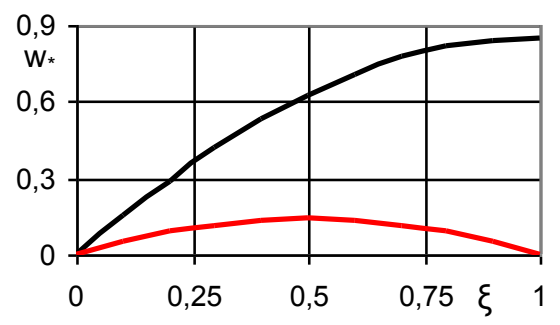

a)

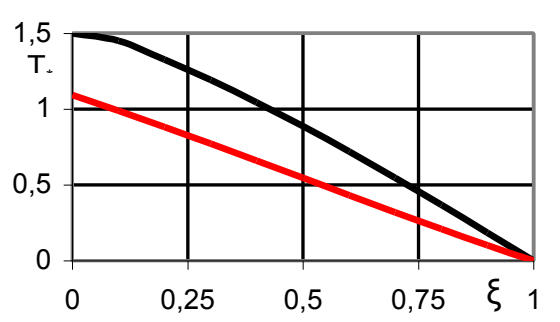

b)

Fig.2. The dependence of (a) the displacement $u_{*}$ and (b) the force $T_{*}$ on the coordinate $\xi$.

The amplitude of vibrations can be further reduced by using cutted electrodes. We divide the length of the beam into two parts $\left[\xi_{0}, \xi_{1}\right],\left[\xi_{1}, \mathbf{1}\right]$. On the segment $\left[\xi_{0}, \xi_{1}\right]$ there is an electrical load $P_{1}$, on the second part $\left[\xi_{1}, \mathbf{1}\right]$ there is an electrical load $P_{2}$. We will find values $P_{1}$ and $P_{2}$ meeting the conditions for the equality of zero displacements at two points $\xi=\xi_{1}$ and $\xi=\mathbf{1}$

$$
\xi=\xi_{1}: \boldsymbol{u}_{*}=\mathbf{0} ; \quad \xi=\mathbf{1}: \boldsymbol{u}_{*}=\mathbf{0}
$$


In the general case, in order to reduce the amplitude of vibrations for any variability of the stress-strain state, we divide the beam into $n$ intervals $\left[\xi_{0}, \xi_{1}\right],\left[\xi_{1}, \xi_{2}\right], \ldots,\left[\xi_{n-1}, \xi_{n}\right], \xi_{0}=\mathbf{0}$, $\xi_{n}=\mathbf{1}$. At each interval on each electroelastic layer there is a pair of cutted electrodes. Denote the electrical load through $P_{i}$ on the interval with number $i$ of the beam. At each interval, we choose such an electrical load that would maximally reduce the amplitude displacements of the beam in this segment.

The unknown electrical load on the interval with number $i$ of the beam has the form

$$
\left.\varphi^{( \pm 2)}\right|_{x_{3}= \pm \boldsymbol{h}}= \pm V_{i},\left.\quad \varphi^{( \pm 2)}\right|_{x_{3}= \pm h_{1}}=\mp V_{i}
$$

We will find unknown quantities $\boldsymbol{P}_{i}(\boldsymbol{i}=\mathbf{1}, \ldots, \boldsymbol{n})$ using the conditions

$$
\xi=\mathbf{0}: \quad u_{*}{ }^{(\mathbf{1})}=\mathbf{0} ; \quad \xi=\xi_{i}: u_{*}{ }^{(i)}=\mathbf{0}, \quad u_{*}{ }^{(i+1)}=\mathbf{0}, \quad T_{i}=T_{i+1} ; \xi=\mathbf{1}: \quad u_{*}{ }^{(n)}=\mathbf{0}, T_{n}=\mathbf{0}
$$

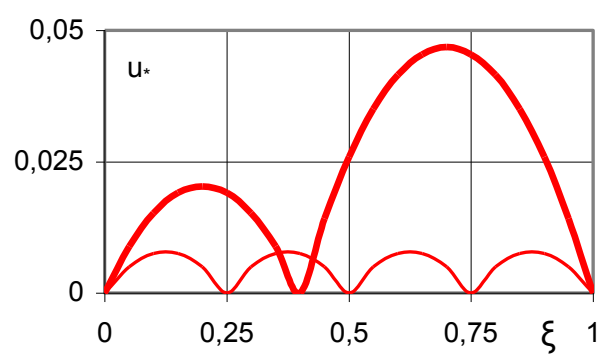

a)

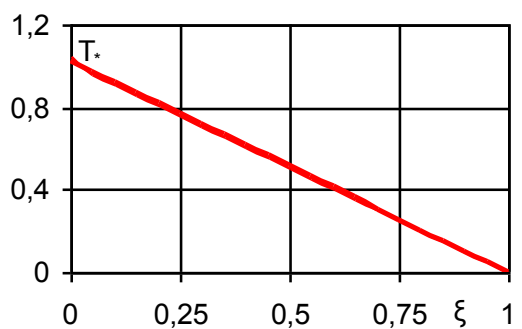

b)

Fig.3. The distribution of a) the displacement $u_{*}$, and b) the force $T_{*}$ along the length of the beam after vibration suppression.

The calculation results for $n=2, \lambda_{1}=\mathbf{1}$ (thick red line) and $n=4, \lambda_{1}=\mathbf{1}$ (thin red line) are presented in Fig. 3. From the graphs it can be seen that with an increase in the number of pairs of electrodes, the amplitude of the displacements decreases rapidly, and the amplitudes of the forces practically do not change.

\section{Transverse vibrations of a three -layer beam}

It is assumed that the following electrical load acts on the beam:

$$
V^{(2)}=-V^{(-2)}=V_{b}
$$

We introduce the dimensionless sought values

$$
\xi=\frac{x}{l}, \boldsymbol{w}_{*}=\frac{\boldsymbol{w}}{l}, \kappa_{*}=\boldsymbol{l} \kappa=\frac{d^{2} w_{*}}{d \xi^{2}}, N_{*}=\frac{l^{2}}{M} N, G_{*}=\frac{l}{M} G, Z_{*}=\frac{l^{3}}{M} Z, Q_{*}=\boldsymbol{Q} \frac{l}{M}
$$

Taking into account the formulas (29), the system of equations (23) will be rewritten as 


$$
\frac{d N_{*}}{d \xi}+Z_{*}-\lambda_{2}^{4} w_{*}=\mathbf{0}, N_{*}=\frac{d G_{*}}{d \xi}, G_{*}=\kappa_{*}-Q_{*}, \kappa_{*}=\frac{d^{2} w_{*}}{d \xi^{2}}
$$

The resolving equation for the bending problem is written as

$$
\frac{d^{4} w_{*}}{d \xi^{4}}-\lambda_{2}^{4} w_{*}+Z_{*}=\mathbf{0}, \lambda_{2}^{4}=-\frac{2 h \rho \omega^{2} l^{4}}{M}
$$

The solution of equation (31) is

$$
\begin{gathered}
w_{*}=c_{1} \operatorname{ch} \lambda_{2} \xi+c_{2} \operatorname{sh} \lambda_{2} \xi+c_{3} \cos \lambda_{2} \xi+c_{4} \sin \lambda_{2} \xi+\frac{1}{\lambda_{2}^{4}} Z_{*} \\
c_{1}=-c_{3}-\frac{1}{\lambda_{2}^{4}} Z_{*}=-\frac{Z_{*}}{\lambda_{2}^{2} \delta}\left(\operatorname{ch} \lambda_{2} \cos \lambda_{2}+\operatorname{sh} \lambda_{2} \sin \lambda_{2}+1\right), \quad c_{2}=-c_{4}=\frac{Z_{*}}{\lambda_{2}^{2} \delta}\left(\operatorname{ch} \lambda_{2} \sin \lambda_{2}+\operatorname{sh} \lambda_{2} \cos \lambda_{2}\right)
\end{gathered}
$$

We calculate the natural frequencies of the beam using the equation

$$
\delta=\mathbf{2}\left(\operatorname{ch} \lambda_{2} \cos \lambda_{2}+\mathbf{1}\right)
$$

The first three natural frequencies are 1.875, 4.694, and 7.855.

Let the beam perform harmonic vibrations with low variability under the action of a normal load. $Z_{*}$ Reduce the amplitude of vibrations of the beam using the unknown electrical load $\boldsymbol{Q}_{*}$. We choose the value $\boldsymbol{Q}_{*}$ in such a way that the largest deflection $w_{*}$ on the free edge of the beam vanishes.

If the stress-strain state has a large variability, a large number of electrode pairs should be used. When $n$ pairs of electrodes are used, we have $n$ segments of the beam $\left[\xi_{0}, \xi_{1}\right],\left[\xi_{1}, \xi_{2}\right], \ldots,\left[\xi_{n-1}, \xi_{n}\right], \xi_{0}=\mathbf{0}, \xi_{n}=\mathbf{1}$. At each section of the beam, the solution will be written as problem

$$
\begin{gathered}
w_{i}=c_{\mathbf{1}}{ }^{(i)} \operatorname{ch} \lambda_{\mathbf{2}} \xi+c_{\mathbf{2}}{ }^{(i)} \operatorname{sh} \lambda_{\mathbf{2}} \xi+c_{\mathbf{3}}{ }^{(i)} \cos \lambda_{\mathbf{2}} \xi+c_{\mathbf{4}}{ }^{(i)} \sin \lambda_{\mathbf{2}} \xi+\frac{\mathbf{1}}{\lambda_{\mathbf{2}}^{\mathbf{4}}} Z_{*} \\
\left.w_{\mathbf{1}}\right|_{\xi=\mathbf{0}}=\mathbf{0},\left.\frac{d w_{\mathbf{1}}}{d \xi}\right|_{\xi=\mathbf{0}}=\mathbf{0},\left.\quad w_{i}\right|_{\xi=\xi_{i}}=\mathbf{0},\left.\quad w_{i+1}\right|_{\xi=\xi_{i}}=\mathbf{0},\left.\quad \frac{d w_{i}}{d \xi}\right|_{\xi=\xi_{i}}=\left.\frac{d w_{i+1}}{d \xi}\right|_{\xi=\xi_{i}}, \quad i=\mathbf{1}, \mathbf{2}, \ldots, n \\
\left.G_{i}\right|_{\xi=\xi_{i}}=\left.G_{i+\mathbf{1}}\right|_{\xi=\xi_{i}},\left.\quad N_{i}\right|_{\xi=\xi_{i}}=\left.N_{i+\mathbf{1}}\right|_{\xi=\xi_{i}},\left.G_{\boldsymbol{n}}\right|_{\xi=\mathbf{1}}=\mathbf{0},\left.\quad N_{n}\right|_{\xi=\mathbf{1}}=\mathbf{0}, \quad i=\mathbf{1}, \mathbf{2}, \ldots, n-\mathbf{1} \\
\xi_{i-\mathbf{1}} \leq \xi \leq \xi_{i}, \quad \xi_{\mathbf{0}}=\mathbf{0}, \quad \xi_{\boldsymbol{n}}=\mathbf{1}, \quad i=\mathbf{1}, \mathbf{2}, \ldots, n
\end{gathered}
$$

The system of algebraic equations contains $5 n$ unknown constants and $5 n$ equations. The results of calculations of the deflection at a) $\lambda_{1}=1$ and b) $\lambda_{1}=\mathbf{4}$ are presented in Fig. 4 . As above, the black line refers to the desired values without vibration suppression, and the red line with vibration suppression. Fig. 4 a) depicts the deflection of a beam with one pair and two pairs of electrodes and Fig. 4 b) depicts the deflection of a beam with four pairs of electrodes. 


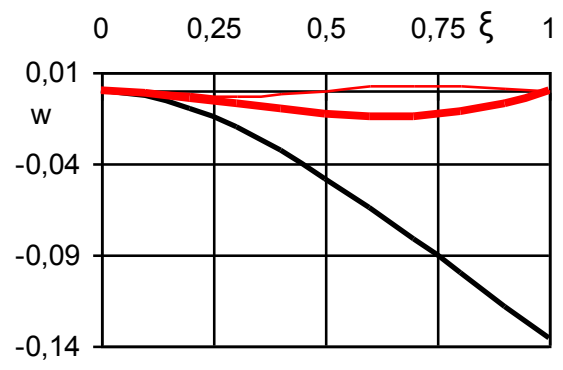

a)

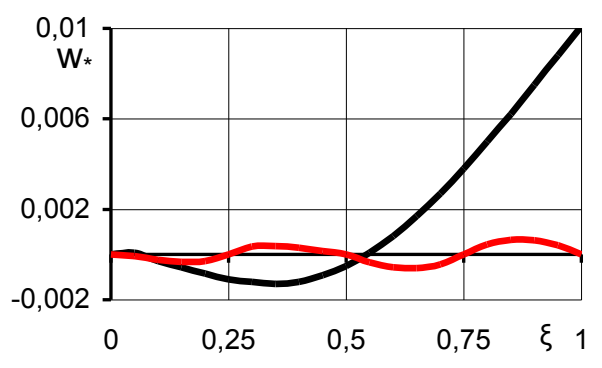

b)

Fig. 4. The distribution of the deflection along the length of the beam at a) $\lambda_{1}=1$ and b) $\lambda_{1}=4$.

A thick red line (Fig. 4 a)) shows that when using one pair of electrodes on each piezoelectric layer, the maximum deflection value decreases 7 times. A thin red line (Fig. 4 a)) shows that when using two pairs of electrodes on each piezoelectric layer, the maximum value of the deflection decreases 40 times. A thick red line (Fig. 4 b)) shows that when using four pairs of electrodes on each piezoelectric layer, the maximum deflection value decreases 10 times.

\section{Conclusions}

Analytical solutions of the problem of beam vibration suppression are obtained. Numerical calculations confirming the effectiveness of using the piezoelectric effect are performed. It is shown that 1) with an increase in the number of electrodes, the efficiency of vibration suppression greatly increases, 2) the greater variability of the electroelastic state, the greater the number of electrode pair that should be used.

\section{References}

1. A. Preumont, Kazuto Seto, Vibration Control of Active Structures (Wiley\&Sons, (Wiley\&Sons, 2008)

2. D. J. Inmah, Vibration with Control. (Wiley Online Books. IBSB: 9781119375081, 2017)

3. D. A. Berlincourt, D. R. Curran, H. Jaffe, Piezoelectric and piezomagnetic materials and their function as transducer. Mason W P(eds.) Physical Acoustics 1A (New York Academic Press, 1964)

4. IEEE Standart on Piezoelectricity, (New York: ANSI-IEEE Std. 176, IEEE, 1987)

5. N. N. Rogacheva, The Theory of Piezoelectric Shells and Plates (Roca Braton: CRC Press, 1994)

6. N. N. Rogacheva, J. of Applied Mathematics and Mechanics, 74(3), 721-734 (ELSEVIER, 2010) 